\title{
Controlling disturbances of islanding in a gas power plant via fuzzy-based neural network approach with a focus on load-shedding system
}

\author{
M. Moloudi ${ }^{1}$ - A. H. Mazinan ${ }^{2}$
}

Received: 21 January 2017 / Accepted: 6 June 2018 / Published online: 15 June 2018

(c) The Author(s) 2018

\begin{abstract}
In a number of power plants such as the South Pars Gas Company, efficient control performance regarding the turbine generator is always needed to avoid facing disturbances of islanding in the supply of power, at each instance of time. It is particularly important to deal with through state-of-the-art techniques, due to the fact that the separation of the electrical grid may increase or reduce the speed of the above-referenced turbine generator and correspondingly the frequency, in its permitted range, may be deviated to stop power generation, abruptly. With this key goal, a set of control strategies through the representation of the gas turbine via Rowen is proposed in the present research. In a word, there are some classical and intelligence-based control approaches to cope with the disturbance of islanding with a focus on load-shedding system. In reality, the proposed investigation is mainly concentrating on the fuzzy-based neural network control approach to cope with the turbulence of frequency deviation. The main contribution made in this research is to deal with gas turbine model in association with generator in conjunction with load-shedding system, while intelligence-based techniques considering fuzzy, neural network and genetic algorithm are synchronously established to design the control strategy with high accuracy. In the sequel, the effectiveness of the proposed intelligence-based control approach is verified, while the classical proportionalintegral-derivative control approach via the Ziegler-Nichols and also the simulated annealing tuning processes are taken as the benchmarks.
\end{abstract}

Keywords Islanding disturbances · Fuzzy-based neural network control approach · Load-shedding system · Gas power plant · Frequency deviation

\section{Introduction}

Up to now, the uninterrupted supply to consumers is known as one of the most important challenges in the area of power industry. In the event of significant customers, paying attention to these challenges is somehow critical. It should be noted that the entire plants with the aim of increasing the stability of the unit are connected to the power grid. Since a

A. H. Mazinan

mazinan@azad.ac.ir; ahmazinan@gmail.com;

ah_mazinan@yahoo.com

1 Department of Control Engineering, South Tehran Branch, Islamic Azad University (IAU), Tehran, Iran

2 Department of Control Engineering, Faculty of Electrical Engineering, South Tehran Branch, Islamic Azad University (IAU), No. 209, North Iranshahr St., P.O. Box 11365/4435, Tehran, Iran variety of reasons such as human error, system error and other related key problems in conjunction with the power grid are provided; it is to note that there is a risk of unintended disconnection to indicate study perturbation effect of unwanted separation is vital. Hence, recommending a solution on that case to eliminate the effect of disturbances or reduce it may be so useful to make and therefore power plant can continue its work for providing electricity, as long as much of important loads remain in network. In the power plant, turbine is connected to a generator, in general. With mechanical torque, it leads to the rotating generator and electric power generation. There is direct relationship between electric production frequency and generator rotation speed. Hereinafter, in proportion to the amount of power that is needed for consumers, it can be applied to the electrical torque generator against the mechanical torque, generated by the turbine. So the speed and its speed variation (acceleration) in the complex turbine generators can be affected by the difference between 
mechanical torque and electrical torque. Since the turbine mechanical torque is related to the amount of injection fuel, the injected fuel quantity is used as a control agent to deal with the mechanical torque. All power plants are normally connected to the power grid.

The island mode operation occurs, as long as a power plant is disconnected due to some reason and therefore there is the biggest challenge in the area of the frequency control to be dealt with. Because if before separating the electrical grid, the required power of consumers is provided exclusively through the grid, after separation from the grid, electric load on the generator suddenly increases and reduces speed rotation of the generator turbine and increasing under frequency. It is in synchronous generators, due to the equation of $N=\frac{120 f}{p}$, where $N$ is taken as the generator rotation speed, $f$ is taken as the frequency of production voltage and finally $p$ is taken as the number of poles of the generator. While the system is in the process of delivering power to the grid prior to occurring the island mode operation, after separation, the speed of rotation of the turbine generator and the corresponding frequency is increased. Since the frequency must be within a specified range, the increased or decreased frequency over its limit leads to aggressive frequency variation. Subsequently, it leads to power plant's departure from network. In this case, the same monopoly power consumers (consumers are not connected to the national grid and are in fact connected directly to the power plant), can lose the power. The South Pars power plant is considered in this research that is provided to supply electric power of the refinery and is connected to stabilize the electrical network. And the island state in a situation that leads to lower frequency, the evaluation of the appropriate control approach to guarantee the frequency stability not out of its range of frequency in the transient state is designed.

\section{The related works}

Given the importance of power system in the island mode operation, a number of potential investigations are carried out in the field of frequency stability and also control design to cope with the frequency and the corresponding voltage $[1,2]$. More than three decades pass of representing the gas turbine by Rowan, which is a linear model that is widely used in academic and industrial environments. Because of the highly nonlinear nature of the gas turbine, a set of state-ofthe-art studies continued to provide more accurate models, although the aforementioned Rowan representation is still known as one of the most well-known models of the gas turbine. The use of soft computing, because of the simplicity and acceptable performance in turbine control areas is usually interesting for researchers in a long time ago [3-12]. It is to note that fuzzy-based techniques are generally taken into consideration as the most important methods in the area of gas turbine control and also in its launch process that have been particularly attended [13-18]. In a word, there are some potential works in this field of gas turbine speed control as well as the study of the frequency variations in line with the amount of power produced, while the performance of classical control approaches are considered. Hereinafter, the intelligence-based approaches including neural network and so on as a way to manage load shedding are studied, in order to guarantee the appropriate performance of the frequency stability [19-22].

The rest of this paper is organized as follows: The proposed control approach is given in Sect. 2. The simulation results are given in Sect. 3 and finally the concluding remarks are provided in Sect. 4, as well.

\section{The proposed control approach}

The proposed control approach is illustrated in Fig. 1, where $P_{\mathrm{p}}$ is taken as the industrial power of plant unit and $P_{\mathrm{n}}$ is taken as the power received in electrical grid. In one such case, $P_{\text {export }}$ that is the sum of the generated and received power from the electrical grid is passed on consumers. At each instance of the island mode operation, the connection with the electrical grid has been cut and as a result, $P_{\mathrm{n}}$ may be zero. Therefore, the power plant, in its alone manner, should produce $P_{\text {export }}$ or in other words should compensate $P_{\mathrm{n}}$. It should be noted that by increasing the aforementioned $P_{\mathrm{n}}$, the intense disturbance of the island mode operation and its control procedure are taken into consideration as more important issues.
Fig. 1 The relationship between power plant, electrical grid and consumers

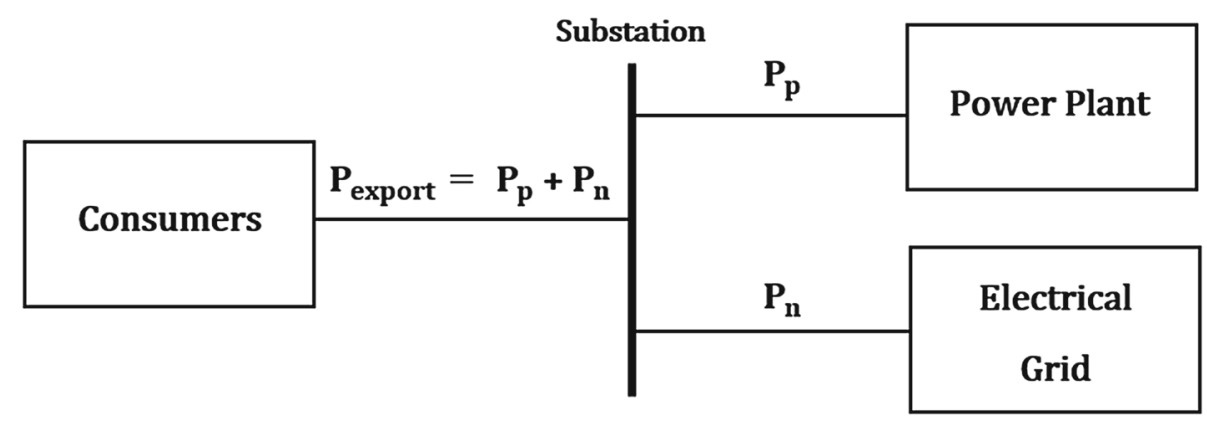


The proposed control approach is designed based upon the proportional-integral-derivative (PID), while its coefficients are all according to the conditions that are determined through the fuzzy-based method. Therefore, in island mode operation, the fuzzy-based system determines the optimal PID parameters. The genetic algorithm is designed to achieve an optimal fuzzy-based system that determines parameters fit with the best situations.

After optimization of the fuzzy-based system, the transient response is not optimal, certainly per critical states. A condition in which before separation of the grid is to receive the significant power, representing the deviated frequency in transition state. Load shedding is used for this purpose to solve the problem as a control solution. In the contribution made here, determining the loss shedding and its amount is performed by a multilayer neural network. Therefore, the key goal is to design and optimize the fuzzy-based PID control approach via the genetic algorithms, in order to achieve optimal response for frequency of generation power in a gas power plant in its transient state of the island operation mode. Now, to guarantee the desired response in all conditions, the load-shedding management system based on the neural network needs to be designed [2,11,13,18,20-22].

\section{The system modeling}

Various models have been introduced regarding the gas turbine systems, where each one of them can provide a representation of the aforementioned turbine system, approximately, with some limitations [7-10]. Turbine and generator are first modeled based upon Rowen linear model $[3,4]$.

\section{Turbine model}

The simplified Rowen linear representation is just illustrated in Fig. 2, ignoring some temperature limitations of turbine [19]. In this model, $T_{\mathrm{L}}$ parameter is taken as the mechanical torque by equipment connected to the turbine, against the torque of the turbine, which enters the turbine shaft. Hereinafter, $T_{\mathrm{e}}$ parameter is taken as the electric torque of generators in power plants, which enters the turbine in the opposite direction, while $T_{\mathrm{d}}$ is taken as the mechanical torque generated by the turbine. In such a case, $W_{\mathrm{f}}$ parameter is taken as the fuel flow and $\mathrm{N}$ is taken as the rotating turbine speed. It is to note that all the input and output parameters in the Rowen model are in per unit. Now, Eq. (1) represents the relation between $T_{\mathrm{d}}, N$ and $W_{\mathrm{f}}$ via functional block $f_{1}$.

$f_{1}=T_{\mathrm{d}}=1.3\left(W_{\mathrm{f}}-0.23\right)+0.5(1-N)$

Since the present research does not consider the use of accurate model of generator, considered the generator as ideal and no casualties, and Eq. (2) is only provided to explain the relation between the active power of generators and corresponding electric torque. It should be noted that the process of controlling the generator is not here considered [22].

$T_{\mathrm{e}}=\frac{P_{\mathrm{e}}}{\omega}$

According to this equation, $T_{\mathrm{e}}$ is taken as the load torque and $P_{\mathrm{e}}$ is taken as the active power produced by the generator, while $w$ is also taken as the angular velocity of generator. Hence, with a focus on this one, by considering the angular velocity and the delivered active power, the electric torque can be calculated. As long as in Rowen model all input and output parameters including $\mathrm{N}$ and $T_{\mathrm{L}}$ have been defined according to per unit, the relationship is applied directly at the end of the turbine model as is now illustrated in Fig. 3. So, the effect of changing the electrical load on the gas turbine load torque is considered in the present model and finally its changes would be effective on the turbine and the generator speed.

\section{The control realizations}

The control realizations in this research are based upon (1) the traditional PID control approach, which is designed in line with Ziegler-Nichols technique and also (2) the intelligencebased control approach. In the sequel, the load-shedding

Fig. 2 The Rowen model [19]

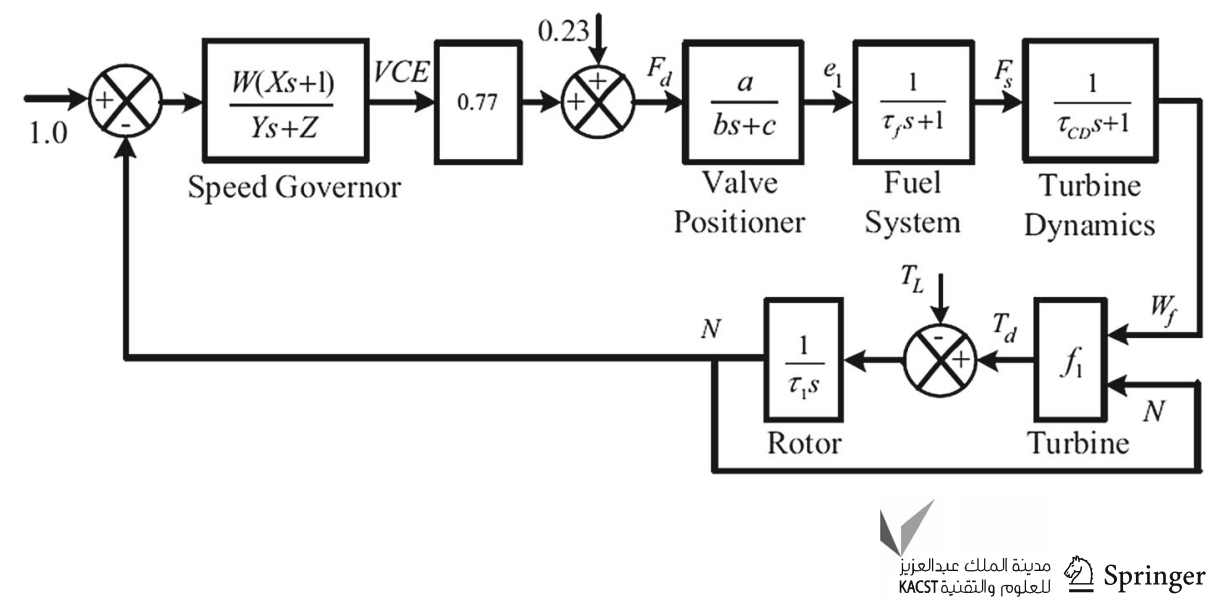




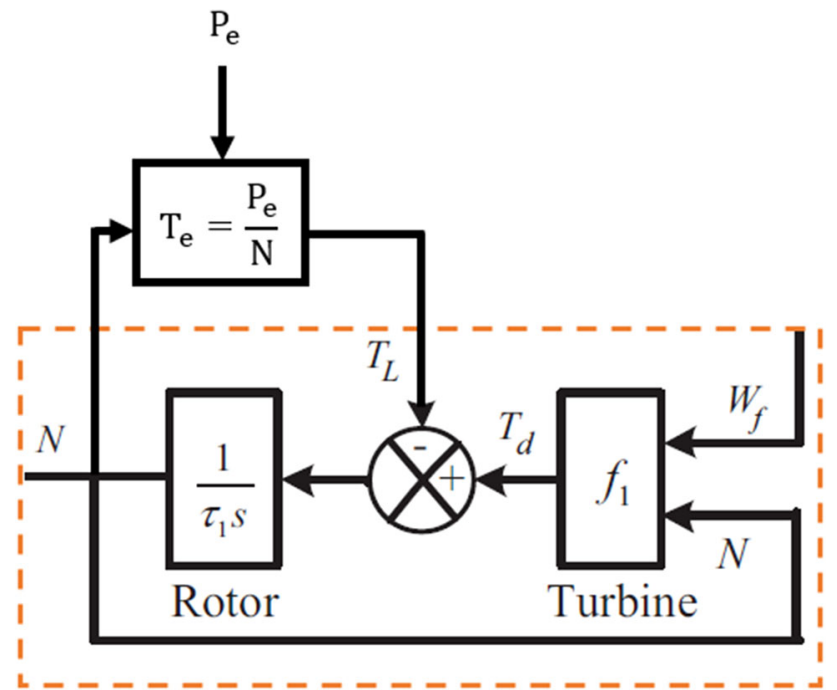

Fig. 3 The Rowen model by considering the torque generators [17]

management system is realized based upon the outcomes of both realizations through the integration of the neural network and the PID control approach. Regarding the classical PID control approach, the coefficients are obtained regarding the Rowan model. According to this traditional control approach, features such as inflexibility, particularly the performance of the control approach is not perfectly acceptable and therefore due to wide use of applications in industries, its upgraded version is presented, in the next sections.

\section{The intelligence-based control approach}

The intelligence-based control approach is illustrated in Fig. 4, where $P_{\mathrm{e}_{1}}$ and $P_{\mathrm{e}_{2}}$ are respectively taken as the amount of power produced by the generator before the separation of the grid and also the amount of power produced by the same generator after separation from the grid. It is to note that the amount of power that is used by consumers is exclusively connected to the power plants, which has some information of system status before the island mode operation.

There are $e$ and $\dot{e}$ as speed error and its derivative. In a word, the responsibility of the fuzzy-based system is to determine the best parameter regarding the PID control approach, at each instant of time.

\section{Realization without load-shedding system}

At first, it is supposed to avoid establishing neural network and load-shedding system in the proposed control approach, illustrated in Fig. 4 and therefore the fuzzy-based control approach is in need of trying to minimize the effect of turbulence. So, before the outbreak of disturbances (separation of electrical network), industrial power of generators can be equal to $P_{\mathrm{e}_{1}}$. And its value, at each instance of separation, increases to $P_{\mathrm{e}_{2}}$. In this situation, the PID parameters are regularly updated via the fuzzy-based system. Thus, the fuzzy-based system with PID control approach can cope with the confusion that has been created due to the occurrence of harmonic distortion in island mode operation. In this way, an attempt to keep the frequency control and prevent excessive loss of frequency can be occurred. As is shown in Fig. 4, the speed error and its derivative have been considered as two inputs of fuzzy-based system. As stated before, the present fuzzy-based system determines the best values for PID control approach parameters in different situations. It should be noted that input membership functions are selected as the triangular function and output membership functions are also selected as Gaussian function. For each input, seven functions including the negative big (NB), the negative medium (NM), the negative small (NS), the zero $(\mathrm{Z})$, the positive small (PS), the positive medium (PM) and finally the positive big (PB) are considered. The present input membership functions are presented in Fig. 5 [15,16].

There are $K_{\mathrm{p}}^{\prime}, K_{\mathrm{d}}^{\prime}$, and $\alpha$ as the outputs of fuzzy-based system, while $K_{\mathrm{p}}^{\prime}, K_{\mathrm{d}}^{\prime}$, are normalized based upon the amounts
Fig. 4 The schematic diagram of the intelligence-based control approach

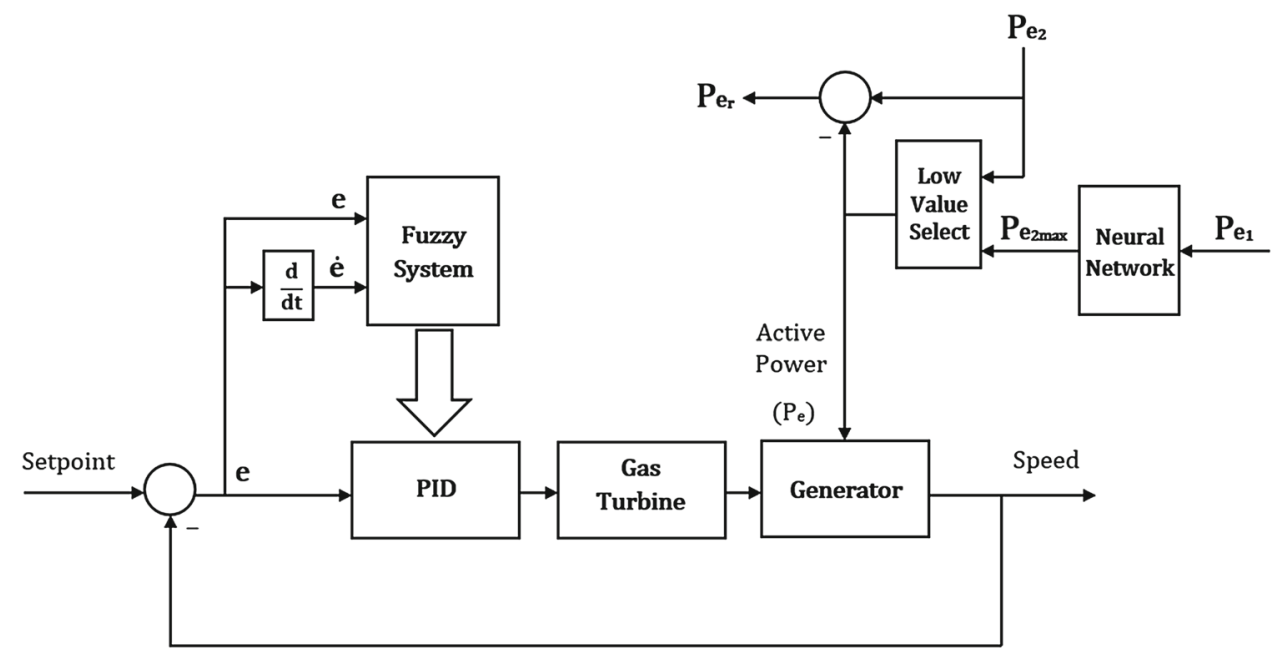




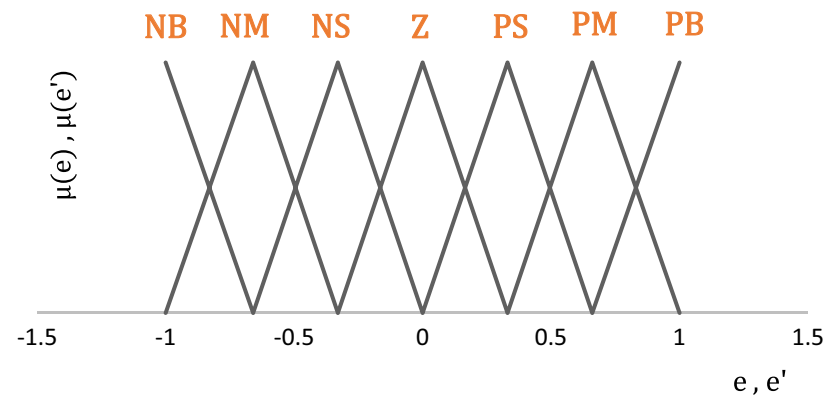

Fig. 5 The input membership functions in the fuzzy-based system

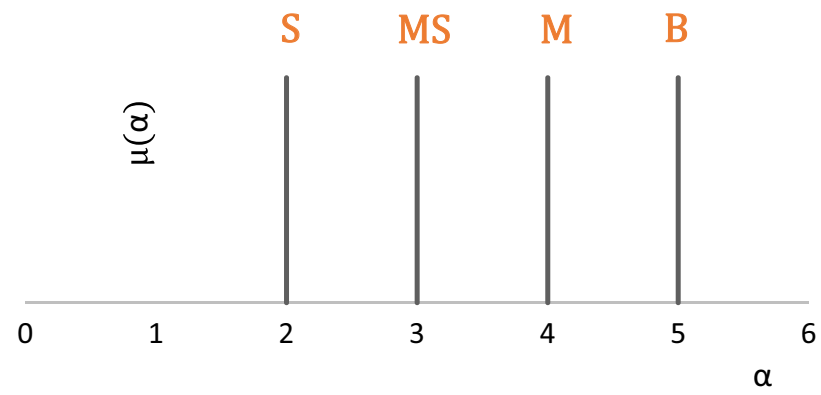

Fig. 6 The membership functions regarding $\alpha$ in the fuzzy-based system

of $K_{\mathrm{p}}$ and $K_{\mathrm{d}}$ in the span of zero to one and $\alpha$ is taken as the proportion between $T_{\mathrm{i}}$ as the constant integrator time and $T_{\mathrm{d}}$ as the constant derivative time [15].

$T_{\mathrm{i}}=\alpha T_{\mathrm{d}}$

Now, with regard to PID control approach, it can write by the following

$K_{\mathrm{i}}=\frac{K_{\mathrm{p}}^{2}}{\alpha K_{\mathrm{d}}}$

The membership functions regarding the $\alpha$ are presented in Fig. 6 and also membership functions regarding the $K_{\mathrm{P}}^{\prime}$ and $K_{\mathrm{d}}^{\prime}$ are illustrated in Fig. 7, as long as two membership functions are taken into consideration as small (S) and big (B), respectively. The entire combinations of both inputs of the fuzzy-based system are here considered through IF and THEN rules. In other words, the present rules are complete to deal with all the input's variations. IF and THEN rules are all determined by genetic algorithm (GA) to provide the optimization procedure [18].

In this research, for simplicity and also providing the remarkable performance, the minimum inference engine in association with the gravity center defuzzification mechanism is designed. It is shown that the minimum required speed, i.e. the frequency is taken to be $99 \%$. In other words, from the instance of separation of the grid, the fuzzy-based

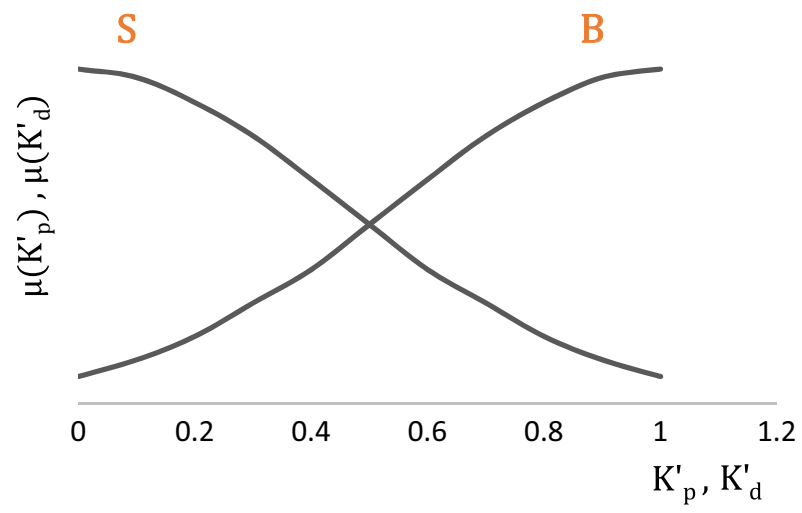

Fig. 7 The output membership functions regarding the $K_{\mathrm{P}}^{\prime}$ and $K_{\mathrm{d}}^{\prime}$ in fuzzy-based system

control approach should somehow deal with the speed to prevent from responding to any point not less than $99 \%$, quickly, and therefore power delivery cannot abruptly be disrupted. Because of the inertia of the turbine as well as the possible difference between $P_{\mathrm{e}_{1}}$ values and $P_{\mathrm{e}_{2}}$, the performance of the proposed control approach may not be desirable, all the time. In general, a set of constraints can be provided to guarantee that the proposed one can always be efficient and accurate. Hence, the speed as the frequency can be less than the minimum amount. By keeping the power plant in the network, or in other words, dealing with the effect of the biggest disturbance as the result of islanding can be taken into account as main goal of the research. In order to complete the presentation of the proposed control approach, illustrated in Fig. 4, it is to note that $P_{\mathrm{e}_{2 \max }}$ is taken as the largest power, where the system could produce in accordance with the amount of $P_{\mathrm{e}_{1}}$, at each instance of separation and the fast transient response is not less than $99 \%$ in no point. In addition, $P_{\mathrm{e}_{\mathrm{r}}}$ is defined as rejected electrical power as load shed at each instance of separation, the neural network output determines it.

\section{Realization with load-shedding system}

In order to realize the proposed control approach with loadshedding system, the previous assumptions of the proposed one are totally taken into account in new connection with the neural network, as illustrated in Fig. 8. Naturally, the difference between the amount of active power produced by power plants and also the amount of power consumed by consumers is higher and therefore the disturbance can be larger.

For cases that disturbance is large the fuzzy-based systems in association with the traditional control approaches do not have the merit of dealing with it, usually. So, for some amounts of $P_{\mathrm{e}_{1}}$ and $P_{\mathrm{e}_{2}}$ that disturbance of separation of them are so great, the designed fuzzy-based control approach may not keep speed on its permitted range. Hereinafter, the responsibility of the load-shedding system is to

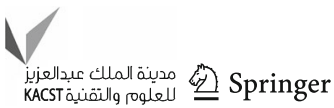




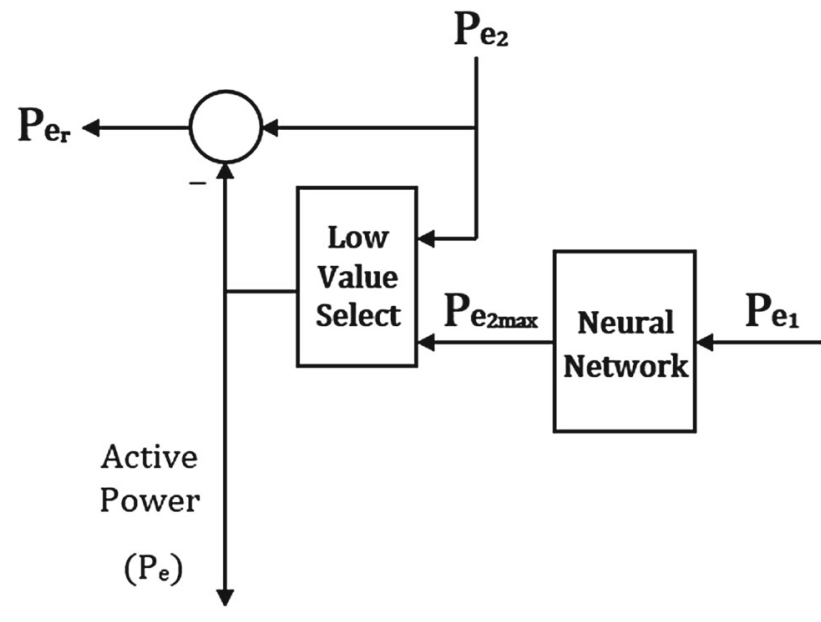

Fig. 8 The control approach with load-shedding system

cope with the disturbance by reducing consumers through the potential fuzzy-based system in association with the corresponding control approach to address the concerns of the frequency deviations to be maintained in its permitted range. With this goal, the neural network should be trained by some responses provided by control approach, obtained per different amounts of $P_{\mathrm{e}_{1}}$ and $P_{\mathrm{e}_{2}}$. It is to note that the sudden increase in electric torque $T_{\mathrm{e}}$ of generator is the main reason of decreasing speed at each instance of separation from the electrical grid. As stated before via Eq. (2), the direct relationship between this torque and the amount of active delivered power of generator depends on the number of consumers. So, for some amounts of $P_{\mathrm{e}_{1}}$ and $P_{\mathrm{e}_{2}}$ that disturbance of separation may be great and the $T_{\mathrm{e}}$ is reduced by load-shedding system, i.e. there is the reduction of $P_{\mathrm{e}_{2}}$, at each instance of separation from the grid. As a result, the control approach is faced a smaller disturbance and can keep the outcomes, in its permitted range. While the optimization of the fuzzy-based system is described in the proceeding section, the control system response for different amounts of $P_{\mathrm{e}_{1}}$ and $P_{\mathrm{e}_{2}}$ can be studied. For each amount of $P_{\mathrm{e}_{1}}$, the largest amount of $P_{\mathrm{e}_{2}}$ is determined and the fuzzy-based control system could keep pace more than 99\% $\left(P_{\mathrm{e}_{2 \max }}\right)$. The attained information is used as instructional data for neural network. With this purpose, the neural network with one input and one output is required. Multilayer perceptron with a hidden layer is the appropriate neural network in this research, as illustrated in Fig. 9 [12].

For the above-referenced neural network training the pair of $P_{\mathrm{e}_{2 \max }}$ and $P_{\mathrm{e}_{1}}$ is used to obtain the speed response through fuzzy-based control approach. The electrical load connected to the generator can be equal to $P_{\mathrm{e}_{2 \max }}$, where $P_{\mathrm{e}_{\mathrm{r}}}$ is taken as the amount of load shedding in Eq. (5).

$P_{\mathrm{e}_{\mathrm{r}}}=P_{\mathrm{e}_{2}}-\min \left(P_{\mathrm{e}_{2 \max }}, P_{\mathrm{e}_{2}}\right)$

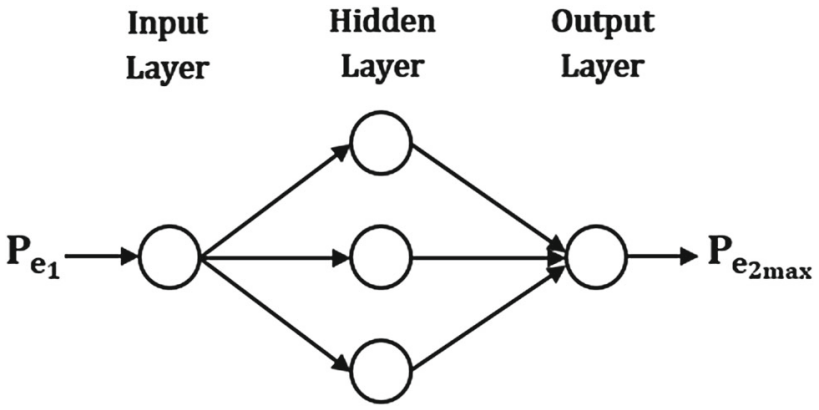

Fig. 9 The complexity of the neural network in load-shedding system

\section{The optimization of fuzzy rules via GA}

The optimization of fuzzy rules is now provided without neural network that means there is no load shedding. So, load power for generator after separation of grid is equal to the amount of consumed power $P_{\mathrm{e}_{2}}$. In this stage, the fuzzy rules are generated for the largest possible confusion, where $P_{\mathrm{e}_{1}}=0$ and $P_{\mathrm{e}_{2}}=1$ are taken as optimized outcomes via the GA. This one is designed to consider the optimal speed and also the variation of $P_{\mathrm{e}}$ in the span of zero to one, in a particular time, i.e. time after enduring response speed to test the quality of responses of each members of the population in the generation regarding the control structure. The key aim in this research is providing the favorable response of speed and particularly the amount of speed not smaller than $99 \%$ after confusion occurrence. So, the small amount of speed error is the most comprehensive one. Hence, the sum of squared errors is finally used as the fitness function.

As it can be presented here, the control approach should provide the suitable and the acceptable performance both before and after the separation from the electrical grid considering error amounts, in the first moment. And also it is before lasting response to be much larger than error amount after disturbance occurrence and therefore the weight coefficient $(w)$ is used, in order to achieve the balanced performance, at all times. The present $w$ is equal to one before disturbance occurrence and also it is equal to ten after disturbance occurrence. Therefore, the sum of squared errors is used as the fitness function via Eq. (6), i.e.

Fitness function $=\sum_{\mathrm{k}=0}^{N-1}\left[w_{(k T)} \cdot \mathrm{e}^{2}(k T) \cdot T\right]$

In this equation, it can write

$W_{(k T)}= \begin{cases}1 & k T<t_{\mathrm{d}} \\ 10 & k T \geq t_{\mathrm{d}}\end{cases}$

where $t_{\mathrm{d}}$ is equal to time of disturbance occurrence. 
Table 1 The neural network training dataset

\begin{tabular}{|c|c|c|}
\hline $\begin{array}{l}\text { Name } \\
\text { Number }\end{array}$ & $P_{\mathrm{e}_{1}}$ & $P_{\mathrm{e}_{2 \max }}$ \\
\hline 1 & 0 & $0.45(\times 98 \%)$ \\
\hline 2 & 0.05 & $0.51(\times 98 \%)$ \\
\hline 3 & 0.10 & $0.55(\times 98 \%)$ \\
\hline 4 & 0.15 & $0.62(\times 98 \%)$ \\
\hline 5 & 0.20 & $0.66(\times 98 \%)$ \\
\hline 6 & 0.25 & $0.72(\times 98 \%)$ \\
\hline 7 & 0.30 & $0.77(\times 98 \%)$ \\
\hline 8 & 0.35 & $0.82(\times 98 \%)$ \\
\hline 9 & 0.40 & $0.88(\times 98 \%)$ \\
\hline 10 & 0.45 & $0.93(\times 98 \%)$ \\
\hline 11 & 0.50 & $0.98(\times 98 \%)$ \\
\hline 12 & 0.55 & $1(\times 98 \%)$ \\
\hline 13 & 0.60 & 1 \\
\hline 14 & 0.65 & 1 \\
\hline 15 & 0.70 & 1 \\
\hline 16 & 0.75 & 1 \\
\hline 17 & 0.80 & 1 \\
\hline 18 & 0.85 & 1 \\
\hline 19 & 0.90 & 1 \\
\hline 20 & 0.95 & 1 \\
\hline 21 & 1 & 1 \\
\hline
\end{tabular}

\section{The simulation results}

In order to consider the performance of the fuzzy-based neural network control approach proposed here, the investigated outcomes are simulated. In one such case, 15 data are used for instruction, where 3 data are used for validation and also three data are used for final test of the neural network. Furthermore, Levenberg-Marquardt training algorithm is realized. Hereinafter, it is expected to reach in some places that its output can be greater than expected amount, i.e. greater than $P_{\mathrm{e}_{2 \max }}$, and due to the speed response in a point to be smaller than $99 \%$ amount. Hence, in accordance with Table 1, in order to train the neural network dealing with in the areas of high sensitivity instead of couples $\left(P_{\mathrm{e}_{1}}, P_{\mathrm{e}_{2 \max }}\right)$, couples $\left(P_{\mathrm{e}_{1}}, 0.98 P_{\mathrm{e}_{2 \max }}\right)$ are used, which aims us to enhance the system reliability. Before the separation of the grid, the electric power of generators $\left(P_{\mathrm{e}}\right)$ is equal to $P_{\mathrm{e}_{1}}$ and at the moment of separation, the load-shedding acts according to Fig. 9. As a result, by considering $P_{\mathrm{e}_{2}}$ to be smaller than $P_{\mathrm{e}_{2 \max }}$, no load shedding occurred and electrical load connected to generator is equal to $P_{\mathrm{e}_{2}}$, while by considering $P_{\mathrm{e}_{2}}$ to be larger than $P_{\mathrm{e}_{2 \max }}$, it means that in case of lack of load shedding, the speed response can be smaller than $99 \%$. Therefore, the load-shedding system is taken to deal with $P_{\mathrm{e}_{2}}-P_{\mathrm{e}_{2 \max }}$.

In reality, before steady speed of the turbine generator sets, there is no load to be applied to the generator and in fact
$P_{\mathrm{e}}$ amount is considered as zero until some moments before steady speed about $10 \mathrm{~s}$. After that, the initial amount $P_{\mathrm{e}_{1}}$ is applied to it and after $15 \mathrm{~s}$ to simulate the separation of the grid, suddenly, $P_{\mathrm{e}}$ should increase to the second amount $P_{\mathrm{e}_{2}}$. In order to verify the proposed control approach in the presence of disturbances, provided from the separation in four different conditions, four signals are considered for $P_{\mathrm{e}}$, as is now illustrated in Fig. 10.

Hereinafter, Figs. 11 and 12 are provided to clear the speed response of the system under control for the Ziegler-Nichols PID control approach with the tuned coefficients that are now presented in Eq. (8) and also the fuzzy-based PID control approach, while $P_{\mathrm{e}}=0$ is taken.

$\left\{\begin{array}{l}K_{\mathrm{p}}=28.0564 \\ K_{\mathrm{i}}=30.1682 \\ K_{\mathrm{d}}=6.5231\end{array}\right.$

Based on the acquired investigated outcomes, it is obvious that the proposed fuzzy-based PID control approach is able to improve the overshoot characteristics and also to provide the better performance. In the following, the speed responses of the system under control are illustrated through Ziegler-Nichols PID control approach and also the fuzzybased neural network control approach in four $P_{\mathrm{e}}$ signals, as long as the load-shedding system is considered as are all illustrated in Figs. 13, 14 and 15, respectively. In these figures, the speed $99 \%$ is considered as index and hence decreasing speed to be lower than $99 \%$ means that the frequency is taken in its permitted range. It should be noted that Fig. 13 is presented as expected to reach with more severe disturbances, i.e. increasing $P_{\mathrm{e}}$ changes in $15 \mathrm{~s}$, where the final speed loss is also higher and based on $99 \%$ index and frequency has been exceeded of its permitted range for four $P_{\mathrm{e}}$ signals. With a focus on the disturbance of the first to third $P_{\mathrm{e}}$ signals, the frequency has exited of the permitted range and in fact the control approach is only able to reject the disturbance of the fourth signal, effectively, as illustrated in Fig. 14. Hereinafter, comparing the corresponding responses acquired through Ziegler-Nichols PID control approach in Fig. 13, the lowest response rate after the occurrence of any disturbance has been improved that is larger, guarantying the better performance of fuzzy-based neural network control approach in the presence of disturbances. It is obvious that frequency, in its permitted range, is remained in the presence of disturbance regarding all $P_{\mathrm{e}}$ signals, as illustrated in Fig. 15. It is to note that the fuzzybased neural network control approach with load-shedding system is able to deal with the entire $P_{\mathrm{e}}$ signals, effectively.

Furthermore, comparing the corresponding responses shown in Fig. 14, it is viewed that due to load-shedding system, the lowest amount of speed response is improved after the occurrence of any disturbance, which indicate the better performance of fuzzy-based neural network control approach. 
Fig. 10 The set of signals for $P_{\mathrm{e}}$ to deal with the islanding simulation

Fig. 11 The speed response of the system under control through Ziegler-Nichols PID control approach in $P_{\mathrm{e}}=0$
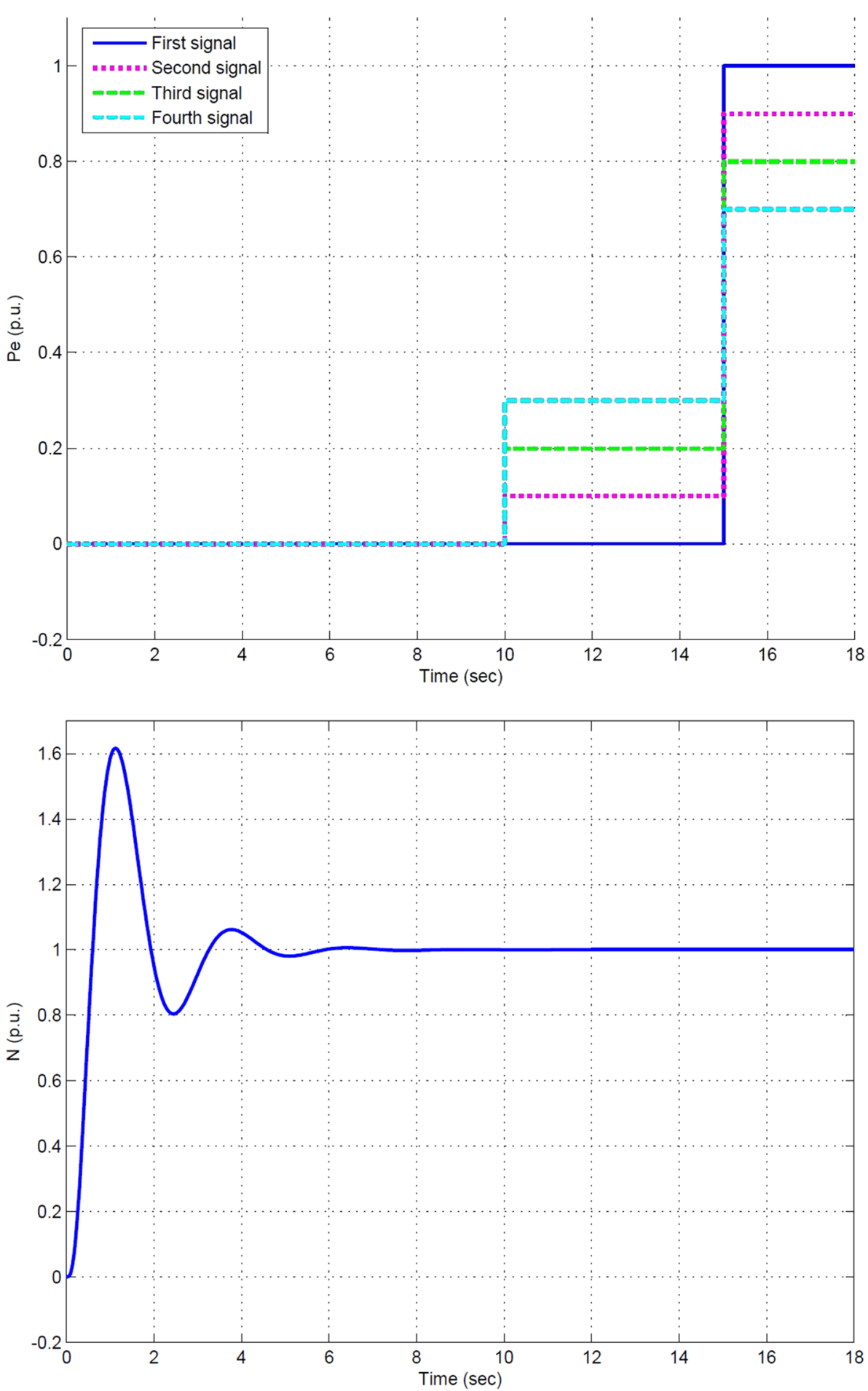

To validate the proposed control approach, which is realized in this research a comparison has been made as tabulated in Table 2. Based on this one, the integral square error (ISE) for instances after the disturbance, i.e. $t \geq 15 \mathrm{~s}$ regarding the first signal $P_{\mathrm{e}}$, as well as the largest possible increase with respect to the initial value $P_{\mathrm{e}}=0$ are correspondingly provided. There is the possibility of dealing with, effectively, the system under control through (1) a set of the classical benchmarks including the PID control approach with the Ziegler-Nichols tuning process and also simulated annealing tuning process, as well as (2) the intelligence-based control approach considering load-shedding system, while the better performance of the second one is clearly guaranteed. 
Fig. 12 The speed response of the system under control through the fuzzy-based neural network control approach in $P_{\mathrm{e}}$ $=0$

Fig. 13 The speed response of the system under control through Ziegler-Nichols PID control approach via four $P_{\mathrm{e}}$ signals
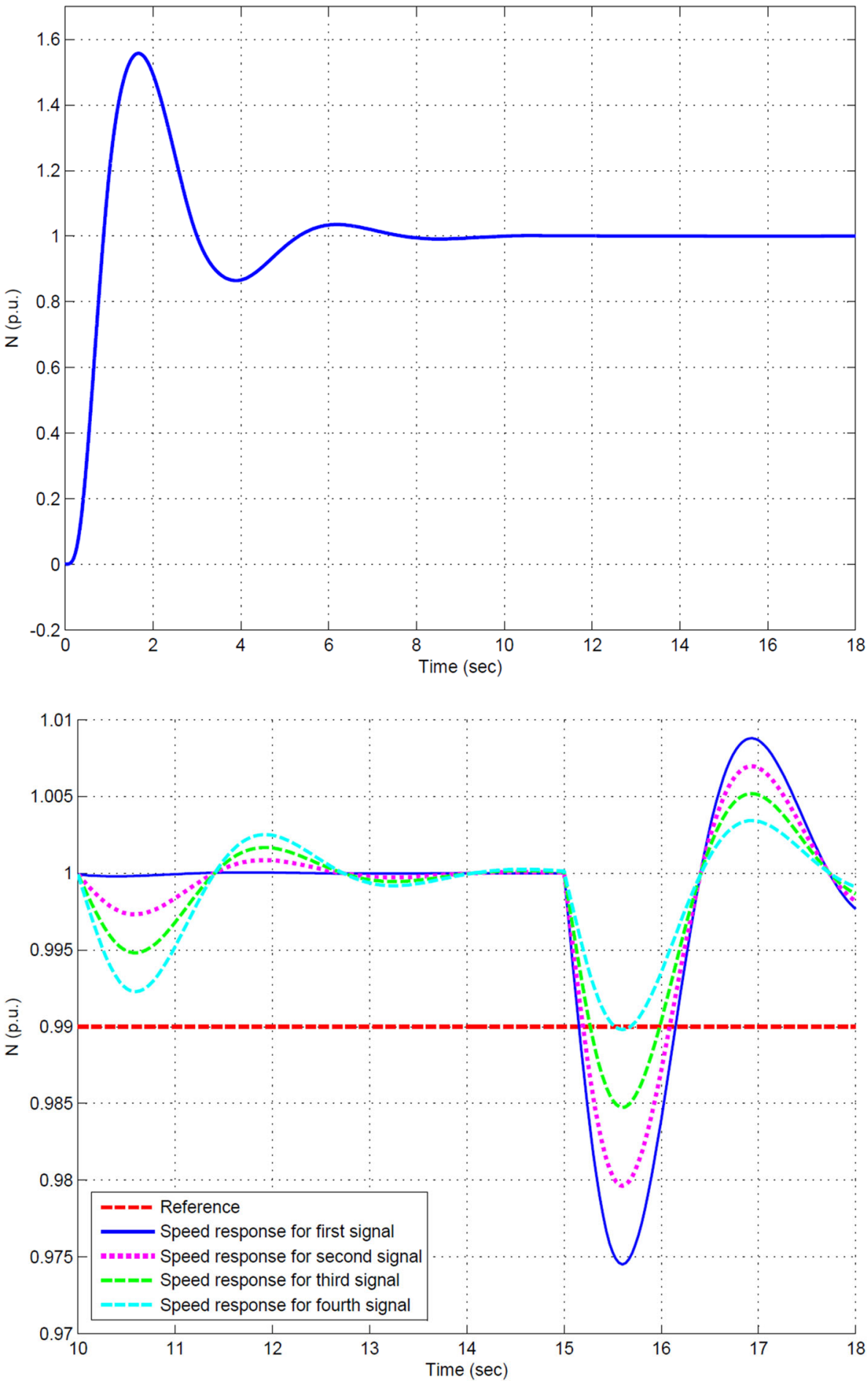

\section{Conclusion}

The present research is investigated to deal with gas power plants via efficient control approach performance to avoid facing disturbances of islanding in the supply of power, at each instance of time. A set of strategies including the classical and the corresponding intelligence-based control through Rowen model are proposed. The main contribution of the proposed idea is made to reject the disturbance of the above-referenced islanding, as long as load-shedding sys- 
Fig. 14 The speed response of the system under control through fuzzy-based neural network control approach via four $P_{\mathrm{e}}$ signals

Fig. 15 The speed response of the system under control through fuzzy-based neural network control approach considering load-shedding system via four $P_{\mathrm{e}}$ signals
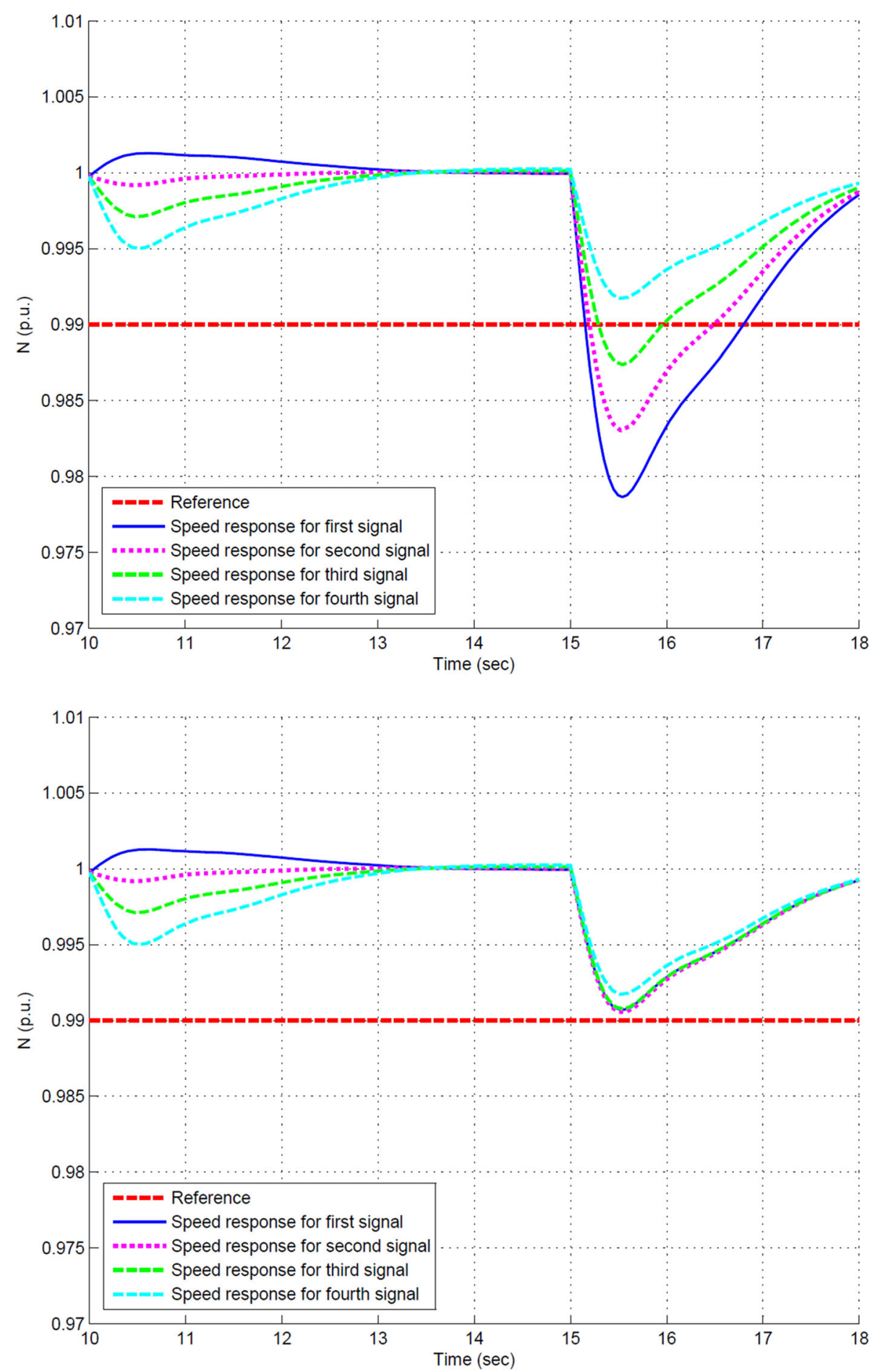

tem is taken into real consideration. In fact, there are the intelligence-based techniques considering fuzzy, neural network and genetic algorithm to be synchronously established, in order to provide potential control approach for guaranteeing high accuracy. The performance of the investigated control approach is finally considered through realizing a set of benchmarks including the classical proportional-integralderivative control approach under the Ziegler-Nichols tuning process and also the simulated annealing tuning process to be correspondingly compared. 
Table 2 The performance of the proposed control approaches with respect to the corresponding benchmarks

\begin{tabular}{lll}
\hline The benchmark and control approaches & The ISE for the largest possible disturbance & The largest allowed increase of $P_{\mathrm{e}}$ \\
\hline The Ziegler-Nichols PID control approach & $4.794 \times 10^{-4}$ & 0.397 \\
The PID control approach, tuned via the simulated annealing & $4.560 \times 10^{-4}$ & 0.423 \\
The fuzzy-based neural network control approach & $4.185 \times 10^{-4}$ & 0.460 \\
$\begin{array}{l}\text { The fuzzy-based neural network control } \\
\text { approach with load-shedding system }\end{array}$ & $9.3856 \times 10^{-5}$ & 1 \\
\hline
\end{tabular}

Open Access This article is distributed under the terms of the Creative Commons Attribution 4.0 International License (http://creativecomm ons.org/licenses/by/4.0/), which permits unrestricted use, distribution, and reproduction in any medium, provided you give appropriate credit to the original author(s) and the source, provide a link to the Creative Commons license, and indicate if changes were made.

\section{References}

1. Baudoin S, Vechiu I, Camblong H (2012) A review of voltage and frequency control strategies for islanded microgrid. In: IEEE international conference on system theory

2. Horne J, Flynn D, Littler T (2004) Frequency stability issues for islanded power systems. In: IEEE power systems conference and exposition

3. Menezes EJN, Araújo AM, Bouchonneau da Silva NS (2018) A review on wind turbine control and its associated methods. J Clean Prod 174:945-953

4. Talaat M, Gobran MH, Wasfi M (2018) A hybrid model of an artificial neural network with thermodynamic model for system diagnosis of electrical power plant gas turbine. Eng Appl Artif Intell 68:222-235

5. Jammu BR, Pati P, Patra SK, Mahapatra KK (2016) FPGA implementation of rule optimization for stand-alone tunable fuzzy logic controller using GA. Complex Intell Syst 2:83-98

6. Coban V, Onar SC (2017) Modeling renewable energy usage with hesitant Fuzzy cognitive map. Complex Intell Syst 3:155-166

7. Yee SK, Milanović JV, Hughes FM (2008) Overview and comparative analysis of gas turbine models for system stability studies. IEEE Trans Power Syst 23:108-118

8. De Mello FP, Ahner DJ (1994) Dynamic models for combined cycle plants in power system studies. IEEE Trans Power Syst 9:16981708

9. Simani S (2005) Identification and fault diagnosis of a simulated model of an industrial gas turbine. IEEE Trans Ind Inform 1:202216

10. Bahrami S, Ghaffari A, Sadati SH, Thern M (2014) Identifying a simplified model for heavy duty gas turbine. J Mech Sci Technol 28:2399-2408

11. Malhotra R, Singh N, Singh Y (2011) Soft computing techniques for process control applications. Int J Soft Comput 3:32-44
12. Bartolini CM, Caresana F, Comodi G, Pelagalli L, Renzi M, Vagni S (2011) Application of artificial neural networks to micro gas turbines. Energy Convers Manag 52:781-788

13. Malhotra R, Singh N, Singh Y (2010) An efficient fuzzy-GA flow control of turbine compressor system: a process control case study. Int J Adv Comput Technol 2:128-139

14. Wang Z, Li S (2009) Simulation study on fuzzy PID control of gas turbine generating sets speed. In: IEEE international conference on intelligent computing and intelligent systems

15. Montazeri-Gh M, Yousefpour H, Jafari S (2010) Fuzzy logic computing for design of gas turbine engine fuel control system. In: IEEE international conference on computer and automation engineering

16. Rodriguez-Martinez A, Garduno-Ramirez R, Vela-Valdes LG (2011) PI fuzzy gain-scheduling speed control at startup of a gasturbine power plant. IEEE Trans Energy Convers 26:310-317

17. Zhao Z, Tomizuka M, Isaka S (1993) Fuzzy gain scheduling of PID control approaches. IEEE Conf Control Appl 23:1392-1398

18. Montazeri-Gh M, Safari A (2011) Tuning of fuzzy fuel control approach for aero-engine thrust regulation and safety considerations using genetic algorithm. Aerosp Sci Technol 15:183-192

19. Balamurugan S, Janarthanan N, Vijaya Chandrakala KRM (2016) Small and large signal modeling of heavy duty gas turbine plant for load frequency control. Electr Power Energy Syst 79:84-88

20. Hsu CT, Kang MS, Chen CS (2005) Design of adaptive load shedding by artificial neural networks. IEEE Conf Gener Transm Distrib 152:415-421

21. Hooshmand R, Moazzami M (2012) Optimal design of adaptive under frequency load shedding using artificial neural networks in isolated power system. Int J Electr Power Energy Syst 42:220-228

22. Sen PC (1997) Principles of electric machines and power electronics. Wiley, New York

Publisher's Note Springer Nature remains neutral with regard to jurisdictional claims in published maps and institutional affiliations. 